\title{
Magnetoresistance signature of resonant states in electromigrated Ni nanocontacts
}

J.-B. Beaufrand, J.-F. Dayen, N. T. Kemp, A. Sokolov, and B. Doudin

Citation: Appl. Phys. Lett. 98, 142504 (2011); doi: 10.1063/1.3576939

View online: https://doi.org/10.1063/1.3576939

View Table of Contents: http://aip.scitation.org/toc/apl/98/14

Published by the American Institute of Physics

\section{Articles you may be interested in}

Novel conducting polymer current limiting devices for low cost surge protection applications Journal of Applied Physics 116, 164501 (2014); 10.1063/1.4899246

Study of molecular spin-crossover complex Fe(phen) $)_{2}(\mathrm{NCS})_{2}$ thin films

Applied Physics Letters 95, 043303 (2009); 10.1063/1.3192355

Ion-assisted deposition of amorphous GaN: Raman and optical properties

Applied Physics Letters 78, 619 (2001); 10.1063/1.1345800

Nonthermal origin of electromigration at gold nanojunctions in the ballistic regime Applied Physics Letters 94, 162103 (2009); 10.1063/1.3124654

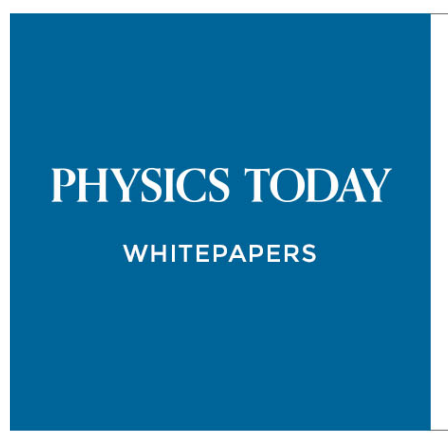

MANACER'S GUIDE

Accelerate R\&D with Multiphysics Simulation

\section{READ NOW}

PRESENTED BY $\checkmark \subset \bigcirc M S \bigcirc L$ 


\title{
Magnetoresistance signature of resonant states in electromigrated Ni nanocontacts
}

\author{
J.-B. Beaufrand, ${ }^{1}$ J.-F. Dayen, ${ }^{1}$ N. T. Kemp, ${ }^{2}$ A. Sokolov ${ }^{3}$ and B. Doudin ${ }^{1, a)}$ \\ ${ }_{1}^{1}$ IPCMS-DMONS, UMR 7504, 23 rue du Loess BP 43, F-67034 Strasbourg Cedex 2, France \\ ${ }^{2}$ Department of Physics, The University of Hull, Cottingham Rd, Kingston Upon Hull HU6 7RX, \\ United Kingdom \\ ${ }^{3}$ Department of Physics and Astronomy, University of Nebraska, Lincoln, Nebraska 68588, USA
}

(Received 11 February 2011; accepted 21 March 2011; published online 6 April 2011)

\begin{abstract}
Fundamental insight is reported into magnetoresistance properties of ballistic-type atomic size Ni nanojunctions obtained at low temperatures. Feedback-controlled electromigration was used to reveal the ballistic nature of the transport and stabilize samples of conductance values in the range of $G_{0}\left(G_{0}=2 e^{2} / h\right)$. Bias voltage dependent measurements identify a clear magnetoresistance fingerprint of resonant tunneling, revealing that localized states in the nanojunctions can be responsible for nonlinear behavior in the IV curves and the related magnetoresistance properties.

(C) 2011 American Institute of Physics. [doi:10.1063/1.3576939]
\end{abstract}

The continuing need for electronics miniaturization makes characterization and control of atomic-sized devices of increasing importance. ${ }^{1}$ This is especially critical for spintronics devices, where the diffusive transport model successfully explaining giant magnetoresistance (MR) should be revisited. Spin transport through few atom contacts has been the subject of intense interest, ${ }^{2}$ motivated by possible ultimate atomic spin valve realization, ${ }^{3}$ with the report of a rich variety of physical phenomena. ${ }^{4,5}$ When decreasing the size of the junction down to a few atoms in size, the related conductance values of a few quanta (multiples of $2 e^{2} / h$ ) is expected to correspond to ballistic-type transport through a few atoms, metallic in nature, and exhibiting anisotropic MR (AMR) properties significantly different from the bulk. ${ }^{6,7}$ After further contact opening, low conductance samples (below $0.1 G_{0}$ ) relates to the evanescent overlap of surface states reaching the tunneling regime of conduction and asymptotically exhibiting tunneling (through vacuum) MR (TMR) behavior when the contact is fully open. ${ }^{8}$ In the intermediate regime of conduction, the situation is more complicated, where quantum interference ${ }^{9}$ and Kondo-Fano resonance can also be invoked in explaining the experimental results. ${ }^{10}$ The complexity and possible overlap or superposition of these different physics make the results interpretations difficult, with discrepancies in the published experimental data revealing variability of properties from sample to sample. This suggests that the magnetoresistive process is sensitive to the local junction environment, such as localized states at the junction interface. ${ }^{11}$

Electromigrated (EM) nanojunctions are obtained by forcing a large current density into a patterned metallic constriction. The related electron wind and Joule heating displace atoms and defects, up to the breaking of the contact. Such samples are particularly suited to studies on magnetic materials, where optimal mechanical stability is needed for limiting electrical transport artifacts caused by magnetostriction and magnetostatic effects. ${ }^{12,13}$ In this letter we report the voltage-controlled feedback fabrication of EM Ni nanojunctions. We follow the evolution of the resistance when slowly

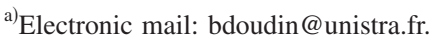

opening the contact, taking advantage of the observed transition from diffusive transport to reveal the occurrence of (quasi) ballistic transport. We focus our discussion to samples of typical conductance value around $G_{0}$, away from the diffusive and tunneling limits.

$\mathrm{Ni}$ and test $\mathrm{Au}$ constrictions were patterned by e-beam lithography and angle deposition $\left(20 \mathrm{~nm}+9.5^{\circ}, 20 \mathrm{~nm}\right.$ $-9.5^{\circ}$ ) in an e-beam evaporator with base pressure of $10^{-7}$ mbar. ${ }^{14}$ The sample was inserted in a vacuum cryostat, with pressure below $10^{-8}$ mbar at temperatures below $10 \mathrm{~K}$. Large current stress was then controlled, in a four-terminal circuit, by a feedback loop (with $1 \mathrm{MHz}$ bandwidth) keeping constant the voltage drop through the junction. This limited the current and Joule heating effects, detrimental to junction stability when reaching very small size ${ }^{15,16}$ Following the procedure of $\mathrm{Wu}$ et al., ${ }^{16}$ we obtained quantitative agreement with their results on Au junctions, not shown here, used as benchmark system to validate the technique. Figure 1(b) shows data from a typical EM measurement process for $\mathrm{Ni}$ samples, where $100 \mathrm{~ms}$ voltage pulses of increasing magnitude are applied through the constriction, while the resistance of the constriction is measured during the jth pulse bias ON $\left(\mathrm{R}_{\mathrm{j}}\right)$ and OFF $\left(\mathrm{R}_{\mathrm{j}}^{0}\right)$ states. We observe a clear change in the electrical transport regime, similar to findings on $\mathrm{Au}$ nanojunctions, ${ }^{16}$ by monitoring the difference between $R_{j}$ and $R_{j}^{0}$. For initially low applied voltages, with closed junctions, we observe $R_{j}$ larger than $R_{j}^{0}$, as expected from heating effects due to scattering [Fig. 1(b)]. When increasing pulse amplitude, a sharp transition to a different heat dissipation mechanism is observed at 200 to $300 \mathrm{mV}$ bias, with $R_{j}^{0}$ and $R_{j}$ significantly increasing but exhibiting approximately the same value. This reveals reduced heating effects of the junction, as expected for the quasiballistic regime, where scattering occurs away from the contact. Reaching that regime facilitates the control of further conductance reduction, down to a fraction of $\mathrm{G}_{0}$. Note that we often observed a small dip before occurrence of the quasiballistic regime. We tentatively interpret this small resistance decrease to a recrystallization effect driven by EM and thermal effects, improving the local resistance of the nanocontact by driving away structural defects. This behavior was observed for the majority of the 
(a)

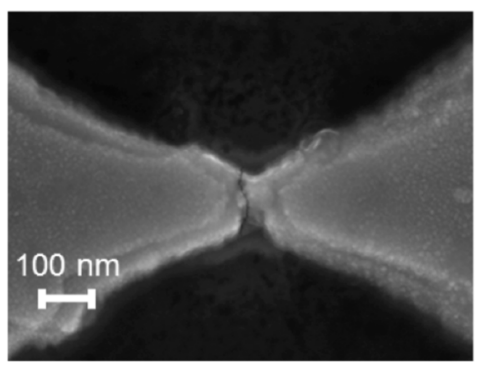

(c)

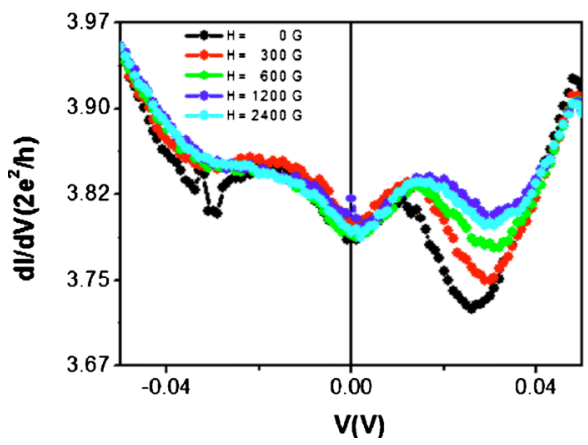

(b)

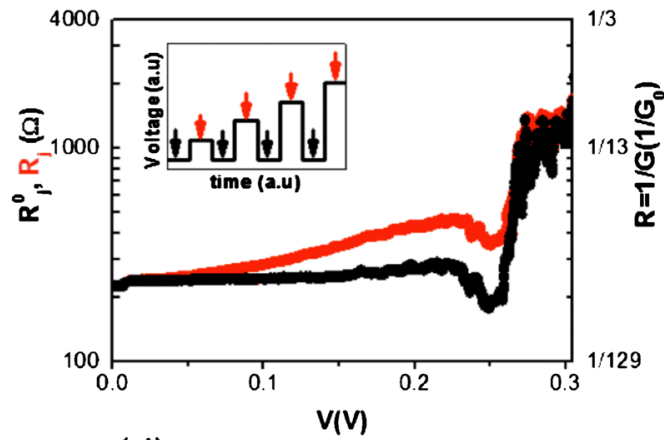

(d)

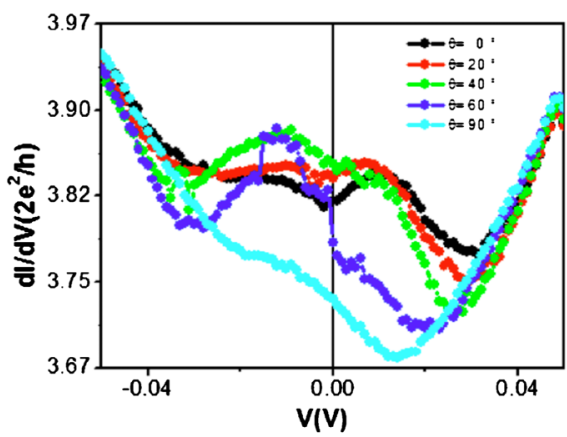

FIG. 1. (Color online) (a) Scanning electron microscopy image of EM Ni junction. (b) Evolution of the Ni nanocontact resistance when applying a $100 \mathrm{~ms}$ voltage pulse of increasing amplitude (inset), measured during "ON" and "OFF" pulse states. (c) Example of voltage bias dependent differential conductance and its variation with magnetic field. (d) Example of voltage bias dependent differential conductance and its variation with angle in a magnetic field of $1 \mathrm{~T}$. samples, but was not systematic. Our success rate for EM in $\mathrm{Au}$ nanocontacts is better than $90 \%$. We found however that $\mathrm{Ni}$ junctions were more prone to thermal runaway, limiting our success rate to approximately $60 \%$. We found it critical to first attain a value of $\mathrm{R}_{\mathrm{j}}^{0}=100-200 \Omega$ before starting our EM procedure (as inspired by Refs. 4 and 5) in order to achieve a success rate better than $90 \%$.

The EM process was pursued until the sample achieved a conductance value of the order of $\mathrm{G}_{0}$. At $10 \mathrm{~K}$, about $15 \%$ of the samples had a few hours stability, robust enough to allow variation in both the magnetic field and the applied DC bias with reproducible results. Typical $d I / d V$ measurements in applied magnetic field for the samples with $3 G_{0}<G$ $<10 G_{0}$ are presented on Figs. 1(c) and 1(d). We observed significantly nonlinear behavior, as similarly reported by Shi et al., ${ }^{9}$ with strong bias-dependence of AMR curves limited to amplitudes below 3\% [Figs. 1(c) and 1(d)].

When decreasing further the conductance of the sample, down to the $0.2 G_{0}<G<3 G_{0}$ range, spin valve-type MR curves were systematically observed, lacking however, sample-to-sample reproducibility. One extensively measured sample is presented in Fig. 2, with MR curves obtained for several voltage biases. The absolute value of MR does not exceed $4.5 \%$, in good agreement with the absence of magnetostrictive effects and confirming the mechanical stability of the junction. The nonuniform shape and significant noise of the magnetoresistive signal is expected and consistent with that of an atomically narrow constricted system. ${ }^{4,5,9}$

Interestingly, a sign dependence of the MR as a function of the dc bias applied can be found, showing a negative signature at $0 \mathrm{~V},+10 \mathrm{mV},+50 \mathrm{mV}$, while becoming positive for $+150,-50$, and $-150 \mathrm{mV}$ (Fig. 2). Such behavior has previously been observed $\mathrm{Ni} / \mathrm{NiO} / \mathrm{Co}$ electrodeposited tunnel junctions, ${ }^{17}$ where the current path was estimated to be limited to filaments a few $\mathrm{nm}$ in size. Tunneling via localized states in the barrier under resonant conditions was
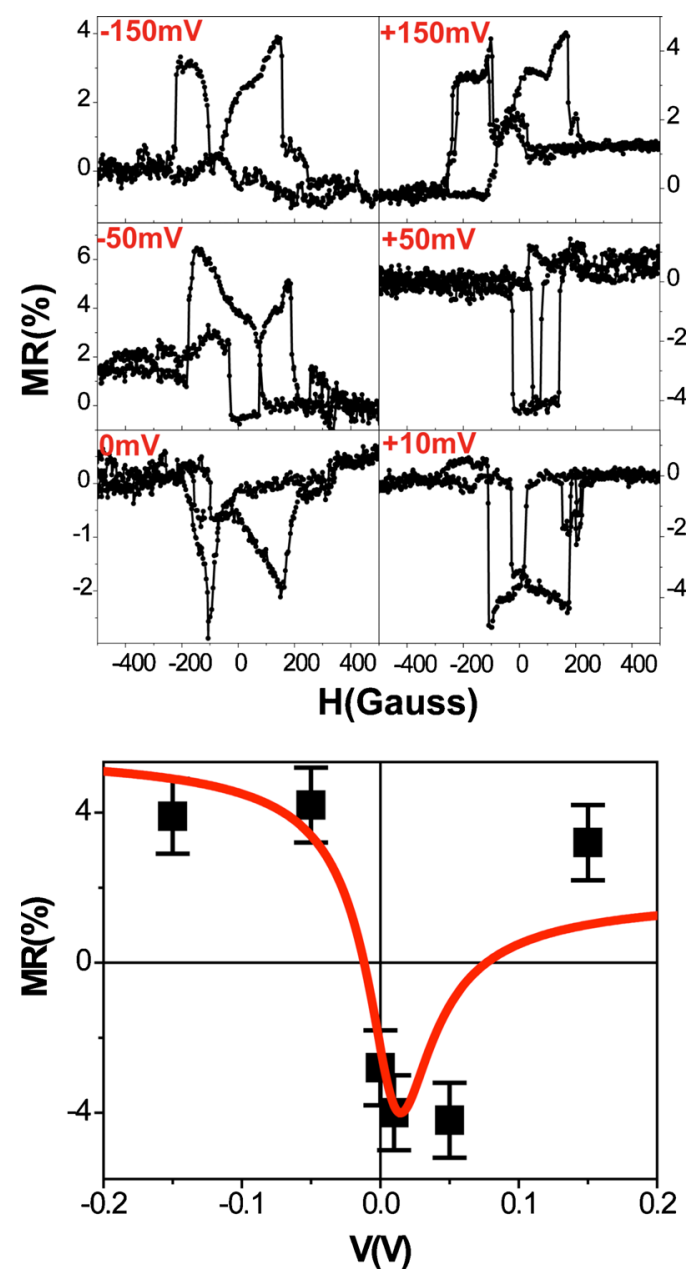

FIG. 2. (Color online) Top: examples of the evolution of MR properties as a function of voltage bias. Bottom: comparison between measured and calculated MR as a function of dc bias applied, using Eqs. (1) and (2). Parameters used are: $E_{i}=10 \mathrm{meV}, \Gamma=7 \mathrm{meV}, \Gamma_{1}=50 \mathrm{meV}, \Gamma_{2}=5 \mathrm{meV}, \mathrm{P}_{1}=\mathrm{P}_{2}$ $=33 \%$. 
proposed as the mechanism for the possible occurrence of change in sign of the effective spin polarization of the conduction electrons. ${ }^{18}$

When charges tunnel from the left to right electrodes, intermediate states in the barrier (due for example to disorder or impurities) can provide a preferred path for transmission if the related energy levels residing within the tunnel barrier become energetically accessible. For tunneling occurring through a localized state of energy $E_{i}$, the conductance per spin channel can be expressed by:

$$
G(E)=\frac{4 e^{2}}{h} \frac{\Gamma_{1} \Gamma_{2}}{\left(E-E_{i}\right)^{2}+\left(\Gamma_{1}+\Gamma_{2}+\Gamma\right)^{2}} .
$$

The total energy broadening $\Gamma_{1}+\Gamma_{2}+\Gamma$ is decomposed as the sum of spin dependent $\Gamma_{1} / h$ and $\Gamma_{2} / h$ leaks to the source and drain magnetic electrodes. Adding a spin independent rate $\Gamma / \mathrm{h}$ relates to spin independent inelastic scattering due to coupling of the resonant state to another degree of freedom such as an electron-electron or electron-phonon interaction. The leak rates are assumed to be of the form $\Gamma_{1}$ $\propto \rho_{1} \exp (-2 \kappa \mathrm{x})$ and $\Gamma_{2} \propto \rho_{2} \exp [-2 \kappa(\mathrm{d}-\mathrm{x})]$, with $\rho_{1}$ and $\rho_{2}$ being the density of states of the left and right electrodes, $\kappa$ the decay constant, and $x$ the position of the impurity state within the barrier of thickness $d$. The spin dependence of the whole system is introduced through the spin polarizations: $P_{1,2}=\left(\rho_{1,2}^{\uparrow}-\rho_{1,2}^{\downarrow}\right) /\left(\rho_{1,2}^{\uparrow}+\rho_{1,2}^{\downarrow}\right)$, where the index 1,2 refers to the source and drain electrodes, and the arrows to the spin of the electron.

As tunneling occurs between two ferromagnetic contacts, spin-dependant leak rates result in conductance values modified by the mutual magnetic alignment of the two electrodes, with associated TMR defined by $\mathrm{TMR}=\left(G_{\mathrm{AP}}\right.$ $\left.-G_{\mathrm{P}}\right) /\left(G_{\mathrm{AP}}+G_{\mathrm{P}}\right)$, where $G_{\mathrm{AP}}$ and $G_{\mathrm{P}}$ being the conductance of the system in the parallel and antiparallel configuration respectively. The TMR value can be predicted as a function of the dc bias $V$ applied, by integrating Eq. (1):

$$
G(V)=\int_{E_{F}}^{E_{F}+e V} G(E) d E .
$$

This simplified model has been shown to provide the key ingredients to spin dependent transport in several heterogeneous systems, where tunnelling is expected to occur through intermediate localized states. ${ }^{18-20}$ It allows in particular simple understanding of the inversion of the TMR, resulting from significant asymmetry in the leak rates $\Gamma_{1} / h$ and $\Gamma_{2} / h$. The data of Fig. 2, chosen as an example of bias-dependent inversion of TMR, is reasonably well explained by this model.

We consider therefore that our findings reinforce the concept of resonance effects in atomic junctions. When breaking a contact, enhanced localization of intermediate states can dominate charge transfer between the two electrodes and bias-dependent energy level alignments can show significant nonlinearity in the current-voltage properties. The striking analogy between data in Fig. 2 and measurements performed on $\mathrm{Ni} / \mathrm{NiO} / \mathrm{Co}$ nanowire junctions ${ }^{18}$ suggest that, in such an ultimately small system, impurity surface states, dopants, disordered structures, and "tip states",21 are possible candidates for the origin of the resonant states. We can also speculate that such a resonance model can be extended to the more conducting and metallic-type contacts (Fig. 1), keeping in mind that the resonant model presented can also be extended to coherent scattering in ballistic transport through atomic constrictions.

In summary, our findings on the MR signature of $\mathrm{Ni}$ nanojunctions supports the model of resonant states providing conduction paths for samples at the onset of contact opening. As illustrated in Ref. 18, resonant tunneling can result in significant statistical dispersion of the observed MR behavior, and can explain the divergent reported results on magnetic nanocontacts. Minimizing these effects should be kept in mind when searching for ultra miniaturized spintronic devices. Lastly, the resonant tunneling model exhibits many similarities to models in single molecule junctions for tunneling through the HOMO-LUMO levels, indicating that atomic-scale spintronics contacts are likely to face the same hurdles as those found for single molecule devices.

We thank the support from the StNano clean room facilities and staff. We acknowledge support from CNRS fellowship (A.S.), and partial support from the Agence Nationale de la Recherche (projects MOSE and SUD) (B.D.) and NSF MRSEC (NSF-DMR Grant No. 0820521) (A.S.).

${ }^{1}$ E. Vogel, Nat. Nanotechnol. 2, 25 (2007).

${ }^{2}$ B. Doudin and M. Viret, J. Phys.: Condens. Matter 20, 083201 (2008).

${ }^{3}$ G. Blumfiel, Nature (London) 426, 110 (2003).

${ }^{4}$ K. I. Bolotin, F. Kuemmeth, A. N. Pasupathy, and D. C. Ralph, Nano Lett. 6, 123 (2006)

${ }^{5}$ Z. K. Keane, L. H. Yu, and D. Natelson, Appl. Phys. Lett. 88, 062514 (2006)

${ }^{6}$ J. Velev, R. F. Sabirianov, S. S. Jaswal, and E. Y. Tsymbal, Phys. Rev. Lett. 94, 127203 (2005).

${ }^{7}$ A. Sokolov, C. Zhang, E. Y. Tsymbal, J. Redepenning, and B. Doudin, Nat. Nanotechnol. 2, 522 (2007).

${ }^{8}$ E. Y. Tsymbal, O. N. Mryasov, and P. R. LeClair, J. Phys.: Condens. Matter 15, R109 (2003).

${ }^{9}$ S.-F. Shi, K. I. Bolotin, F. Kuemmeth, and D. C. Ralph, Phys. Rev. B 76, 184438 (2007).

${ }^{10}$ M. Reyes Calvo, J. Fernández-Rossier, J. J. Palacios, D. Jacob, D. Natelson, and C. Untiedt, Nature (London) 458, 1150 (2009).

${ }^{11}$ J. D. Burton, R. F. Sabirianov, J. P. Velev, O. N. Mryasov, and E. Y. Tsymbal, Phys. Rev. B 76, 144430 (2007).

${ }^{12}$ M. Gabureac, M. Viret, F. Ott, and C. Fermon, Phys. Rev. B 69, 100401 (2004)

${ }^{13}$ W. F. Egelhoff, Jr., L. Gan, H. Ettedgui, Y. Kadmon, C. J. Powell, P. J. Chen, A. J. Shapiro, R. D. McMichael, J. J. Mallett, and T. P. Moffat, J. Magn. Magn. Mater. 287, 496 (2005).

${ }^{14}$ H. Park, A. K. L. Lim, A. P. Alivisatos, J. Park, and P. L. McEuen, Appl. Phys. Lett. 75, 301 (1999).

${ }^{15}$ M. L. Trouwborst, S. J. van der Molen, and B. J. van Wees, J. Appl. Phys. 99, 114316 (2006).

${ }^{16}$ Z. M. Wu, M. Steinacher, R. Huber, M. Calame, S. J. van der Molen, and C. Schönenberger, Appl. Phys. Lett. 91, 053118 (2007).

${ }^{17}$ B. Doudin, G. Redmond, S. E. Gilbert, and J.-P. Ansermet, Phys. Rev. Lett. 79, 933 (1997).

${ }^{18}$ E. Y. Tsymbal, A. Sokolov, I. F. Sabirianov, and B. Doudin, Phys. Rev. Lett. 90, 186602 (2003).

${ }^{19}$ S. Sahoo, T. Kontos, J. Furer, C. Hoffmann, M. Gräber, A. Cottet, and C. Schönenberger, Nat. Phys. 1, 99 (2005).

${ }^{20}$ C. Barraud, P. Seneor, R. Mattana, S. Fusil, K. Bouzehouane, C. Deranlot, P. Graziosi, L. Hueso, I. Bergenti, V. Dediu, F. Petroff, and A. Fert, Nat. Phys. 6, 615 (2010).

${ }^{21}$ A. L. Vázquez de Parga, O. S. Hernán, R. Miranda, A. Levy Yeyati, N. Mingo, A. Martín-Rodero, and F. Flores, Phys. Rev. Lett. 80, 357 (1998). 